\title{
Lack of Sensitivity of Primary Fanconi's Anemia Fibroblasts to UV and lonizing Radiation
}

\author{
Reinhard Kalb, ${ }^{a}$ Michael Duerr, ${ }^{a}$ Matthias Wagner, ${ }^{a}$ Sabine Herterich, ${ }^{a}$ Michaela Gross, ${ }^{a}$ Martin Digweed, ${ }^{b}$ Hans Joenje, \\ Holger Hoehn ${ }^{a}$ and Detlev Schindler ${ }^{a, 1}$

\begin{abstract}
${ }^{a}$ Department of Human Genetics, University of Wuerzburg, Biozentrum, Am Hubland, D-97074 Wuerzburg, Germany; ${ }^{b}$ Department of Human Genetics, Humboldt University, Charite-Campus Virchow, D-13353 Berlin, Germany; and ' Department of Human Genetics, Free University, NL-1081 BT Amsterdam, The Netherlands
\end{abstract}

Kalb, R., Duerr, M., Wagner, M., Herterich, S., Gross, M., Digweed, M., Joenje, H., Hoehn, H. and Schindler, D. Lack of Sensitivity of Primary Fanconi's Anemia Fibroblasts to UV and Ionizing Radiation. Radiat. Res. 161, 318-325 (2004).

Clinical observations and theoretical considerations suggest some degree of radiosensitivity in Fanconi's anemia (FA), but experimental evidence remains controversial. We tested the sensitivity of primary skin fibroblast cultures from all known FA complementation groups to ionizing radiation and ultraviolet light using conventional cell growth and colony formation assays. In contrast to previous studies, and because FA fibroblasts grow and clone poorly at ambient oxygen, we performed our sensitivity tests under hypoxic cell culture conditions. Fibroblast strains from healthy donors served as negative controls and those from patients with ataxia telangiectasia (AT) and Cockayne syndrome (CS) as positive controls. We observed interstrain variation but no systematic difference in the response of FA and non-FA control fibroblasts to ionizing radiation. After exposure to UV radiation, only complementation group A, G and D2 strains displayed values for colony formation $\mathrm{EC}_{50}$ that were intermediate between those for the negative and positive controls. Because of considerable interstrain variation, minor alterations of the response of individual FA strains to ionizing and UV radiation should be interpreted with caution and should not be taken as evidence for genotype-specific sensitivities of primary FA fibroblasts. All together, our data indicate neither systematic nor major sensitivities of primary FA fibroblast cultures of any complementation group grown under hypoxic cell culture conditions to ionizing or UV radiation. ๑ 2004 by Radiation Research Society

\section{INTRODUCTION}

Fanconi's anemia (FA) is an autosomal recessive disease caused by mutations in at least seven different genes (1). Like the ATM, WRN and XP genes, the FA family of genes

\footnotetext{
${ }^{1}$ Correspondence and reprint requests should be addressed to: Department of Human Genetics, University of Wuerzburg, Biozentrum, Am Hubland, D-97074 Wuerzburg, Germany; e-mail: schindler@ biozentrum.uni-wuerzburg.de.
}

belongs to the group of caretaker genes whose presumptive function is to safeguard the genome against endogenous and exogenous DNA damage. Mutations in FA genes cause genomic instability, cell cycle alterations, bone marrow failure and neoplasia. Allogenic bone marrow transplantation (BMT) is the treatment of choice for patients with a matching sibling donor, and the success rate using HLAcompatible unrelated donors is improving (2). While it is well established that FA fibroblasts are highly sensitive to DNA-crosslinking agents such as diepoxybutane, mitomycin $\mathrm{C}$ (MMC) and nitrogen mustard, it remains controversial whether FA cells are sensitive to ionizing radiation (recently reviewed in ref. 3). Within the context of conditioning regimens prior to BMT, there has been a longstanding clinical impression of increased radiosensitivity of FA patients because of prominent skin reactions (4). In contrast, no excess radiation toxicity was observed after 6 Gy singledose total-body irradiation in a recent study of FA patients in a dose escalation trial (5).

In vitro studies with FA cells have also yielded conflicting results, with some studies claiming enhanced sensitivity and others normal sensitivity to ionizing radiation (for review, see ref. 3). To explain this discrepancy, it is conceivable that the various types of assays used to assess radiation sensitivity measure different facets of the radiation response. In addition, radiosensitivity may vary as a function of complementation group since the FA phenotype can be caused by mutations in any one of multiple FA genes. A study by Carreau et al. (6) tested FA lymphoblasts and suggested increased sensitivity of FA complementation groups D2 (EUFA202), E (EUFA410), F (EUFA121), G (EUFA143, EUFA316) and H (EUFA173) to the radiomimetic drug bleomycin. These results were partly at variance with the reassignment of the FA-H line to group FA-A (7), which did not show increased sensitivity. Other evidence regarding potential X-ray (or bleomycin) and UV-radiation sensitivity of FA cells came from studies of the Chinese hamster ovary (CHO) mutant lines UV40 8 and NM3 (9), both of which have genetic defects in the Chinese hamster homologue of the human FANCG/XRCC9 gene. Differenc- 
es in genetic background and the possibility of additional mutations in rodent cell lines derived by chemical mutagenesis may explain these obvious variations in the spectrum of sensitivity of FA and FA-like cells.

Recently, the FANCD2 gene was identified as a putative key player in the FA gene network (10). Exposures to both ionizing and UV radiation were shown to activate a timeand dose-dependent conversion, through monoubiquination, of the FANCD2-S to the FANCD2-L protein, leading to increased numbers of FANCD2 foci in irradiated HeLa cells (10). Moreover, in response to ionizing radiation, FA cells derived from multiple complementation groups failed to show the FANCD2-S to L conversion (10). These studies therefore suggest a biochemical basis for the putative radiation sensitivity of FA cells and implicate the participation of FA proteins in DNA repair pathways.

To further clarify the issue of in vitro sensitivity of human FA cells to ionizing and UV radiation, we undertook a systematic study using primary fibroblast cultures of all known FA complementation groups. A technical aspect that sets the current study apart from previous studies is the fact that cell growth and colony formation after exposure to ionizing and UV radiation was determined in hypoxic rather than ambient oxygen cell culture conditions. Close to physiological hypoxic culture conditions were chosen because of the known oxygen dependence of chromosomal aberrations in FA cells (11), and because FA fibroblasts grow and clone poorly at ambient oxygen, whereas near normal proliferation and colony formation can be achieved under hypoxic culture conditions (12).

\section{MATERIALS AND METHODS}

\section{Cells}

Primary FA fibroblasts were subcultures of reference strains established by the participating laboratories with informed consent from patients undergoing diagnostic evaluation for FA. Assignment to a given complementation group was by complementation by somatic cell hybrids (13) and/or detection of one or two mutant alleles in a given FA gene. Multiple independent strains were obtained for the complementation groups FAA, FA-C and FA-G, whereas only single strains were available for the groups FA-B, FA-D1, FA-E, FA-F and FA-H [which has been reassigned to FA-A (7)]. Two strains were established from siblings belonging to group FA-D2. Two types of control cells were used: negative controls from healthy laboratory volunteers and positive controls from patients with known sensitivity to UV and ionizing radiation. The following 36 strains were investigated in this study [with the corresponding FA mutations shown in parentheses (14)]: complementation group FA-A: EUFA432 (FANCA 3639delT/3639delT), EUFA598 (FANCA exon43del/ exon43del), EUFA517 (FANCA IVS40+1del18bp/n.d.), EUFA880 (FANCA 2493insC/n.d.); group FA-B: HSC230; group FA-C: 77RD325 (FANCC 322delG/1806insA), EUFA001 (FANCC 322delG/322delG), VU811 (FANCC 322delG/322delG), VU911 (FANCC 322delG/322delG), F97/16 (FANCC 322delG/n.d.), F99/194 (FANCC: 322delG/n.d.); group FA-D1: EUFA423; group FA-D2: VU202 and VU008 (both FANCD2 $904 \mathrm{C} \rightarrow \mathrm{T} / 958 \mathrm{C} \rightarrow \mathrm{T})$; group FA-E: EUFA622 (FANCE IVS5-8g $\rightarrow \mathrm{a} /$ IVS5-8g $\rightarrow \mathrm{a})$; group FA-F: EUFA121 (FANCF $16 \mathrm{C} \rightarrow \mathrm{T} / 349-395 \mathrm{del})$; group FA-G: FA1BER (FANCG IVS2 $+1 \mathrm{~g} \rightarrow \mathrm{a} / 346-347 \mathrm{delCA})$, FA15BER (FANCG IVS13-1g $\rightarrow$ c/IVS13-1g $\rightarrow$ c), EUFA316 (FANCG $313 \mathrm{G} \rightarrow \mathrm{T} / 1183-1192 \mathrm{del}), \quad \mathrm{F} 99 / 112 \quad($ FANCG $\quad 313 \mathrm{G} \rightarrow \mathrm{T} / \mathrm{n}$.d.); former group FA-H, reassigned to FA-A (7): EUFA173 (FANCA 2852G $\rightarrow$ A/ exon17-31del). Control fibroblast strains from healthy individuals were F86/15, F88/75, F96/1, F96/35, F98/JRR, F99/9 and F99/GRS. Positive controls with proven hypersensitivity to UV radiation, derived from patients with Cockayne syndrome (CS) included GM00739, GM01428 and GM01098 (NIGMS Human Genetic Mutant Cell Repository, Camden, $\mathrm{NJ})$. Fibroblast strains with proven increased sensitivity to ionizing radiation (strains F95/20, F95/47, F96/35SYM, F96/49SND and F98/ $72 \mathrm{KSA}$ ) were established in the participating laboratories from patients with ataxia telangiectasia (AT). Aliquots of each culture were frozen at in vitro passages 2-3 and were thawed for the present series of experiments. All strains tested negative for mycoplasma at the time of the study.

\section{Reagents and Equipment}

For cell culture, NUNC plasticware (Gibco BRL, Karlsruhe, Germany) was used. Minimum essential medium (MEM) with Earle's salts (catalog no. 41500), pretested fetal bovine serum (FBS; catalog no. 10270), phosphate-buffered saline (PBS; catalog no. 14200), and trypsin-EDTA (catalog no. 35400) were all from the same supplier (Gibco BRL) and were used throughout the study to maintain consistent culture conditions.

Other reagents included MMC (mitomycin 2, medac, Hamburg, Germany) and the Mycoplasma PCR ELISA kit (Roche, Mannheim, Germany).

Ionizing radiation was from a ${ }^{60} \mathrm{Co}$ source. The radiation energy was $1.033 \mathrm{MeV}$ and was distributed evenly through $1 \mathrm{~cm}$ acrylic glass covering the culture flasks The focus-source distance was approximately 80 $\mathrm{cm}$ to yield a dose rate of about $1 \mathrm{~Gy} / \mathrm{min}$. Dosimetry ensured exact dose application. UV radiation of $254 \mathrm{~nm}$ wavelength was from a UV lamp with variable aperture and exposure timer (Type 3.260 002; Schuett, Goettingen, Germany). The dose was adjusted using a UV-C meter (Kuehnast, Waechtersbach, Germany).

\section{Cell Culture and Irradiation Procedures}

Cells were maintained at $37^{\circ} \mathrm{C}$ and $95 \%$ air $/ 5 \% \mathrm{CO}_{2}$ in Earle's minimal essential medium (MEM) supplemented with $16 \%$ FBS in high-humidity incubators equipped with $\mathrm{CO}_{2}$ and $\mathrm{O}_{2}$ sensors (Heraeus, Hanau, Germany). Air was replaced by nitrogen to obtain a final oxygen concentration of $3 \%(\mathrm{v} / \mathrm{v})$. Fibroblasts were grown in $25-\mathrm{cm}^{2}$ tissue culture grade flasks until they reached subconfluence (monolayer of spindle-shaped cells without visible mitotic activity and without crowding). At this stage, the cells were detached using $1 \times$ trypsin-EDTA in PBS. Aliquots of the single-cell suspensions were counted in a Fuchs-Rosenthal chamber. Density of the cell suspension was adjusted to $50,000 / \mathrm{ml}$ in MEM.

For exposure to ionizing radiation, volumes containing 55,000 cells each $(1.1 \mathrm{ml})$ were transferred into seven $1.8-\mathrm{ml}$ plastic cryotubes. While one vial was left untreated, the other six were exposed to $\gamma$ radiation at $0.5,1.0,1.5,2.5,4.0$ and $8.0 \mathrm{~Gy}$. Immediately thereafter, cell suspensions were diluted by a factor of 10 (55,000 cells per $11 \mathrm{ml}$ of medium). Aliquots of $3 \times 0.1 \mathrm{ml}$ from each of the seven vials corresponding to 3 $\times 500$ cells were seeded in three petri dishes $6 \mathrm{~cm}$ in diameter for colony formation studies. The remaining $10.7 \mathrm{ml}$ containing 53,500 fibroblasts was plated in a $25-\mathrm{cm}^{2}$ tissue culture flask (at about 2,000 cells per $\mathrm{cm}^{2}$ ) and incubated for the 5-day growth study. After 5 days, these cultures were suspended by trypsinization and the total number of cells per culture flask $(n)$ was determined using a Fuchs-Rosenthal chamber. The number of population doublings achieved after 5 days (PD) and was calculated using the function $\mathrm{PD}=\log (n: 53,500): \log 2$.

Colony formation was evaluated by plating triplicate aliquots of 500 fibroblasts each in three $60-\mathrm{mm}$ petri dishes containing a total of $5 \mathrm{ml}$ culture medium. There were two medium changes on days 5 and 10 during the 12- to 14-day incubation period. These conditions were chosen in accordance with the studies of Pomp et al. (15) showing the influence of cell density on the outcome of colony-forming assays. At days 12 to 14 after seeding, colonies were simultaneously fixed and stained using $1 \%$ crystal violet in $20 \%$ ethanol for $3 \mathrm{~min}$. The number of colonies was 
TABLE 1

Sensitivity of FA and Control Skin Fibroblast Cultures to Ionizing Radiation, Expressed as $\mathbf{E C}_{50}$ Values

\begin{tabular}{lccccccc}
\hline & \multicolumn{3}{c}{ Cell growth } & & \multicolumn{3}{c}{ Colony formation } \\
\cline { 2 - 3 } \multicolumn{1}{c}{ Group } & $n$ & $\mathrm{EC}_{50} \pm 1 \mathrm{SD}(\mathrm{Gy})$ & $t$ & & $n$ & $\mathrm{EC}_{50} \pm 1 \mathrm{SD}(\mathrm{Gy})$ & $t$ \\
\hline Normal ("negative") controls & 4 & $4.33 \pm 1.22$ & & & 5 & $1.51 \pm 0.31$ & \\
Positive controls (AT) & 3 & $1.45 \pm 0.51$ & 0.023 & & 3 & $0.51 \pm 0.07$ & 0.002 \\
FA-A & 3 & $3.71 \pm 0.58$ & 0.527 & & 4 & $1.82 \pm 0.48$ & 0.325 \\
FA-C & 4 & $4.89 \pm 2.53$ & 0.741 & & 3 & $1.59 \pm 0.40$ & 0.775 \\
FA-G & 4 & $3.67 \pm 0.47$ & 0.070 & & 4 & $1.55 \pm 0.27$ & 0.836 \\
\hline
\end{tabular}

Notes. Means $\pm 1 \mathrm{SD}$ of $\mathrm{EC}_{50}$ values were computed by fitting to dose-response curves reflecting 5-day cell growth in mass cultures and 12- to 14-day colony growth. AT $=$ ataxia telangiectasia; FA-A, FA-C and FA-G $=$ Fanconi's anemia complementation groups A, C and G.

assessed visually by counting and marking the colonies on a light-table projector. All colonies visible by eye were counted by two independent investigators. Eighty to $90 \%$ of these colonies consisted of more than 30 cells per colony. The agreement between the two independent colony counts was better than $95 \%$.

To study the effect of UV radiation, aliquots of 53,500 cells were plated in a single dish and triplicate aliquots of 500 fibroblasts each were seeded in three $60-\mathrm{mm}$ diameter dishes containing $5 \mathrm{ml}$ of culture medium. Cells were allowed to settle overnight before the lid and the culture medium were removed so that cells were exposed for the duration of the irradiation process with no medium over them. This procedure was necessary because culture medium and polystyrol plasticware absorb almost all the UV light and prevent its effective application to the cells. After exposure to UV light, growth medium was restored and changed for the colony formation assay at days 5 and 10. Evaluation of cell growth and colony formation after UV-light exposure was as described for the experiments with ionizing radiation.

\section{Statistics}

The cell and colony counts after irradiation were expressed relative to those for nonirradiated cultures. $\mathrm{EC}_{50}$ values were computed by curve fitting to the corresponding dose-response graphs. Wilson's $t$ test was used to compare fluctuations between FA and control strains.

\section{RESULTS}

Table 1 shows the average $\mathrm{EC}_{50}$ values after $\gamma$ irradiation of cultures representing FA complementation groups A, C and $\mathrm{G}$ for which multiple independent strains were tested. The difference between normal control and AT cells was significant in both types of assay (cell growth and colony formation). In contrast, there was no significant difference in the growth and cloning parameters of all three categories of FA cultures and the (negative) controls. This finding also holds for most comparisons between FA and control cultures that were exposed to UV radiation (Table 2). However, the mean $\mathrm{EC}_{50}$ values for colony formation of the FA$A$ and FA-G strains but not the FA-C strains were intermediate between those of the negative and positive (CS) control cells. Does this observation indicate increased UVradiation sensitivity of fibroblasts belonging to the FA-A and FA-G complementation groups?

\section{Sensitivity to UV Radiation}

The question of differential sensitivity to UV radiation as a function of complementation group can be answered by inspection of Fig. 1A, which displays colony formation as a function of UV-radiation dose for all strains tested. Differences in $\mathrm{EC}_{50}$ values are reflected by the shapes of the dose-response curves indicating a slightly higher sensitivity of the FA-A and FA-G strains to UV radiation compared to the negative controls. This also holds for a single FA-C strain, but there is considerable interstrain variation within all three groups. In contrast, the positive control strains with known sensitivity to radiation (CS) are clearly set apart from FA and negative control cultures. There is

TABLE 2

Sensitivity of FA and Control Skin Fibroblast Cultures to UV Light, Expressed as $\mathrm{EC}_{50}$ Values

\begin{tabular}{lcccccccc}
\hline & \multicolumn{3}{c}{ Cell growth } & & \multicolumn{3}{c}{ Colony formation } \\
\cline { 2 - 3 } \multicolumn{1}{c}{ Group } & $n$ & $\mathrm{EC}_{50} \pm 1 \mathrm{SD}\left(\mathrm{J} / \mathrm{m}^{2}\right)$ & $t$ & & $n$ & $\mathrm{EC}_{50} \pm 1 \mathrm{SD}\left(\mathrm{J} / \mathrm{m}^{2}\right)$ & $t$ \\
\hline Normal ("negative") controls & 3 & $11.06 \pm 1.32$ & & & 3 & $8.19 \pm 0.92$ & \\
Positive controls (CS) & 3 & $1.45 \pm 0.49$ & 0.001 & & 3 & $1.35 \pm 0.24$ & 0.001 \\
FA-A & 3 & $11.10 \pm 1.07$ & 0.976 & & 4 & $5.77 \pm 1.06$ & 0.045 \\
FA-C & 4 & $11.26 \pm 2.54$ & 0.922 & & 6 & $8.42 \pm 2.46$ & 0.893 \\
FA-G & 4 & $10.05 \pm 1.94$ & 0.539 & & 4 & $5.98 \pm 0.87$ & 0.040 \\
\hline
\end{tabular}

Notes. Means $\pm 1 \mathrm{SD}$ of $\mathrm{EC}_{50}$ values were computed by fitting to dose-response curves reflecting 5-day cell growth in mass cultures and 12- to 14-day colony growth. CS = Cockayne syndrome; FA-A, FA-C and FA-G = Fanconi's anemia complementation groups A, C and G. 

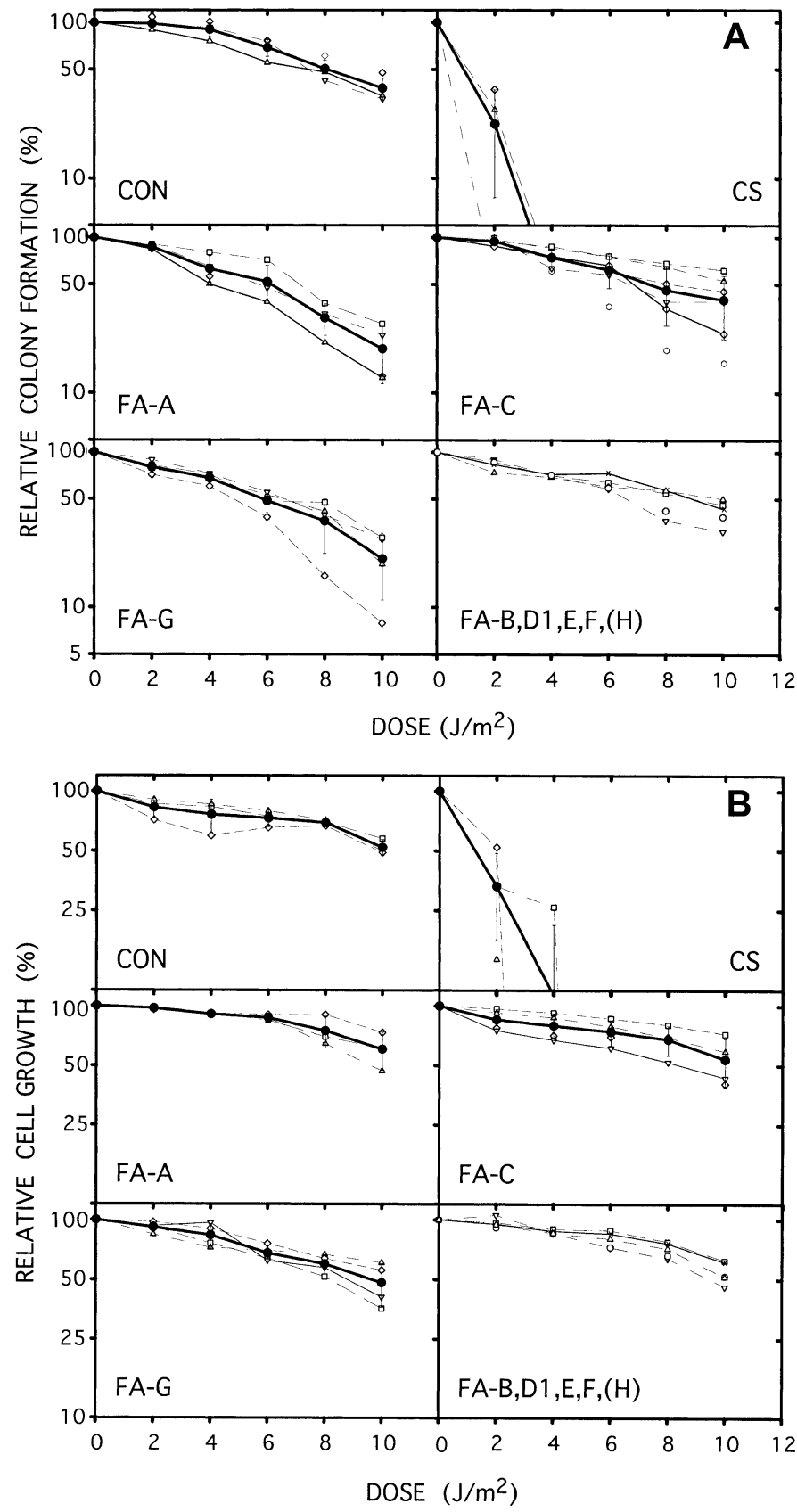

FIG. 1. Panel A: UV-radiation-induced reduction of colony formation. Various types of fibroblasts were exposed to increasing doses of UV light. CON, fibroblast cultures derived from clinically normal individuals (negative controls); CS, fibroblast cultures derived from patients with Cockayne syndrome (positive controls); FA-A, FA-C, FA-G and FA$\mathrm{B}, \mathrm{D} 1, \mathrm{E}, \mathrm{F}, \mathrm{H})$, fibroblast cultures of FA complementation groups $\mathrm{A}-\mathrm{G}(\mathrm{H})$, where $\mathrm{H}$ has been reassigned to A (7). Solid lines with dark circle symbols denote means $\pm 1 \mathrm{SD}$ derived from three to six single curves each (light lines). For complementation groups with only one available strain each, the following symbols are used: circle: FA-B; inverted triangle: FAD1; cross: FA-E; square: FA-F; upward triangle: FA-(H). Panel B: UVradiation-induced reduction of 5-day cell growth. Various types of fibroblasts were exposed to increasing doses of UV light. Abbreviations and symbols used for FA-B,D1,E,F,(H), are as in panel A. no obvious systematic difference in UV-radiation response between FA fibroblasts and those from healthy donors, and all of the FA strains, but none of the CS cells, were able to form colonies at exposures above $4 \mathrm{~J} / \mathrm{m}^{2}$. Figure 1B shows the corresponding data for the 5-day cell growth experiment in mass cultures. As in the colony assay data, there is considerable interstrain variation, but there is no systematic difference between any of the FA groups of strains and the negative controls.

\section{Sensitivity to Ionizing Radiation}

As shown in Fig. 2A, there was a certain amount of interstrain variation within each complementation group as a function of $\gamma$-ray dose, but the degree of this variation was very similar among all the groups tested. In contrast, there was a systematic difference between the dose-response curves for AT cells and all the other (FA and negative control) strains. Both interstrain variation and the difference between FA and AT strains were even more pronounced when 5-day cell growth data were taken as the end point (Fig. 2B). A single FA-G strain appeared to be slightly sensitive to $\gamma$ radiation, albeit much less than the positive (AT) control curves. AT strains stopped growing beyond $2.5 \mathrm{~Gy}$, whereas the relative growth of the exceptional FA-G strain still amounted to $30 \%$ at $4 \mathrm{~Gy}$.

\section{Radiation Response of FA-D2 Fibroblasts}

Since the recently identified $F A N C D 2$ gene appears to play a central role in the function of the FA proteins (1), and since (SV-40 transformed) FA-D2 fibroblasts have been reported to be sensitive to ionizing radiation (10), we paid special attention to fibroblast cultures derived from two siblings belonging to FA complementation group D2. Figure 3 compares the growth and cloning performance of the two D2 strains with the control group means. There was no difference between the $\gamma$-radiation responses of the FA-D2 strains and the non-FA controls. After exposure to UV light, colony formation of the FA-D2 strains declined more steeply than that of the controls, whereas the 5-day cell count assays were similar to those of the non-FA controls. Since the outcome of the colony assay after UV-light exposure was at variance with the 5-day cell count data, the colony formation experiment was repeated three times with identical results.

\section{DISCUSSION}

In analogy to the sensitivity of bacteria to antibiotics, we refer here to radiosensitivity as any enhanced cytotoxic, cytostatic or anti-proliferative reaction to ionizing radiation or UV light. Other types of assays assess the reaction to ionizing radiation or UV light by measuring short-term biochemical effects such as DNA fragmentation, activation of FANCD2-L protein, and the formation of nuclear foci (3, 10). The conditions employed in the present study mainly 
reflect long-term cellular effects of ionizing radiation or UV light. Using multiple primary fibroblast cultures from all known FA complementation groups and comparing their UV-light and ionizing radiation response relative to positive and negative controls, we arrived at the following conclusions:

With AT cells serving as positive controls, there is no evidence for a systematic ionizing radiation sensitivity of FA fibroblasts of any complementation group under the conditions of our assays. Rather, FA fibroblasts exhibit the same dose-response relationship and the same degree of interstrain variation as non-FA control cultures derived from healthy donors. A considerable range in the radiation response of human diploid fibroblast cultures appears to be the rule rather than the exception in this type of study (16, 17). Differences related to cell type, culture history, culture conditions, nutrient supply, and donor genotype may account for such heterogeneity (18-20).

Likewise, our study provides no evidence for increased radiosensitivity of FA-D2 fibroblast cultures derived from two sibling donors. This finding seems to be at variance with the original report on the function of the FANCD2 gene in which SV40-transformed skin fibroblast-derived lines were found to react abnormally to ionizing radiation (10). The apparent discrepancy is unexpected, since transformation of human diploid fibroblasts using either SV40 T-antigen or the HPV16 E6 gene usually results in enhanced rather than decreased radioresistance (21-24). One possible explanation might be that this is the first study in which cell growth and colony formation were assessed under hypoxic culture conditions that mimic in vivo oxygen tension. Cell cycle progression of primary but not transformed FA cells is known to be sensitive to ambient oxygen $(11,12,25)$, and many studies make a case for a protective function of the FA gene products against reactive oxygen species (26). Since damage induced by ionizing radiation is thought to occur in part through the formation of reactive oxygen intermediates (27), hypoxic cell culture conditions might prevent or lessen the burden of such damage in FA cells. This would imply that there is no gross and systematic deficiency of the recognition and/or repair of ionizing radiation-induced damage in FA cells as long as these cells are kept under culture conditions that are hypoxic relative to ambient air but physiological with respect to the in vivo state.

With CS cells serving as positive controls, there is no consistent evidence for increased UV sensitivity of FA fibroblasts. This is uniformly true if 5-day cell growth in mass cultures is taken as the end point. Compared to nonFA control cultures, FA-A and FA-G strains show reduced colony formation at higher doses of UV radiation. Since FANCG/XRCC9 is the human homologue of the gene defective in the UV-radiation-sensitive Chinese hamster ovary cell lines UV40 and NM3 $(8,9,28)$, the marginal UVradiation sensitivity of our FA-G strains is interesting. However, the degree of UV-radiation sensitivity displayed
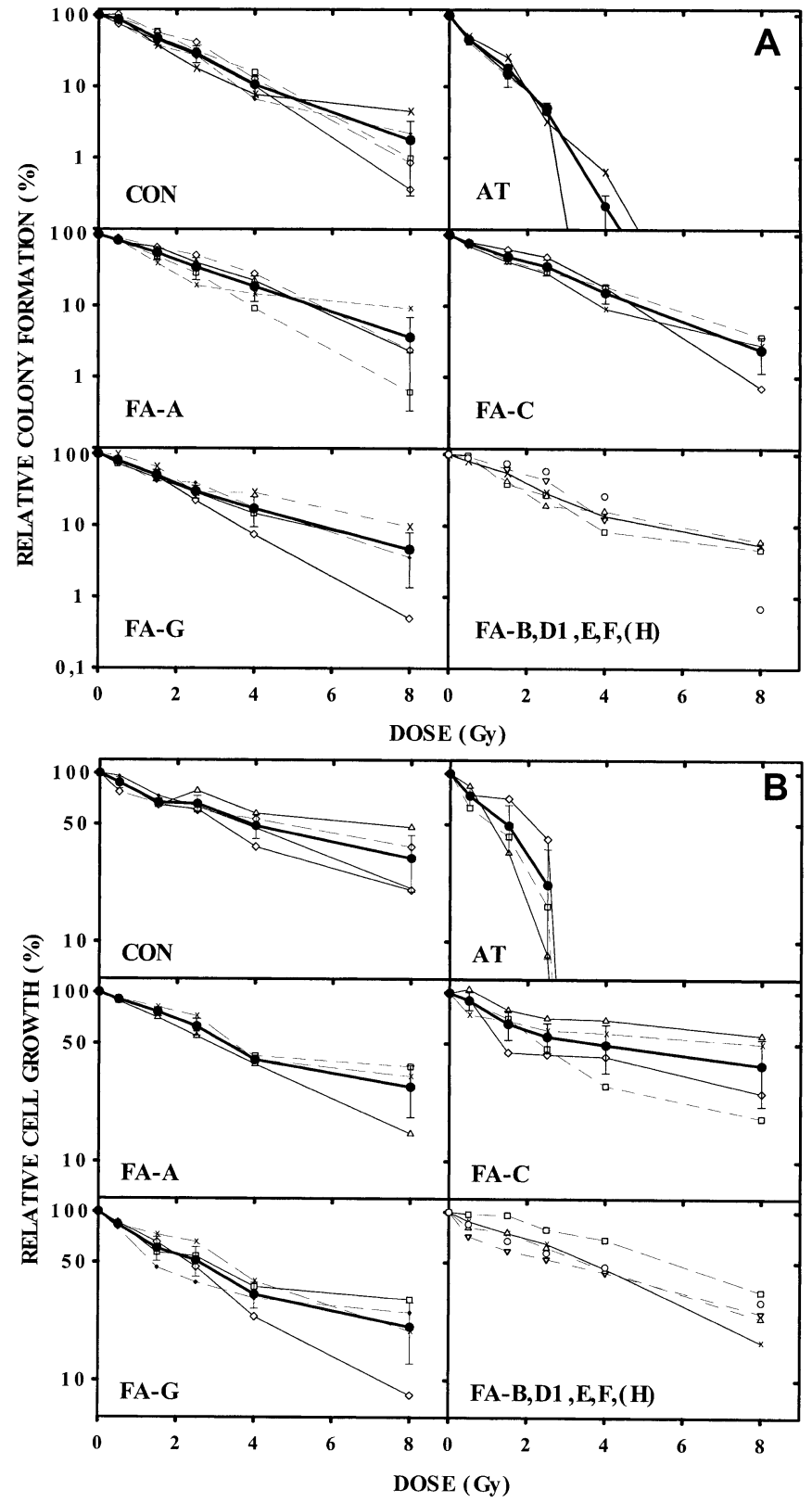

FIG. 2. Panel A: Gamma-radiation-induced reduction of colony formation. Various types of fibroblasts were exposed to increasing doses of ionizing radiation. AT, fibroblast cultures derived from patients with ataxia telangiectasia (positive controls). Other abbreviations, and symbols used for FA-B,D1,E,F,(H), strains are as in Fig. 1A. Panel B: Ionizing radiation-induced reduction of cell growth. Various types of fibroblasts were exposed to increasing doses of ionizing radiation. AT, fibroblast cultures derived from patients with ataxia telangiectasia (positive controls). Other abbreviations, and symbols used for FA-B,D1,E,F,(H), are for complementation groups with only one available strain each, the following symbols are used: circle: FA-B; inverted triangle: FA-D1; cross: FA-E; square: FA-F; upward triangle: FA-(H).

by our group of FA-G strains is in no way close to the sensitivity displayed by the positive control (CS) fibroblasts. In addition, there is considerable interstrain variation, with individual strains within the FA-A and FA-G groups showing a completely non-FA control-like response. 


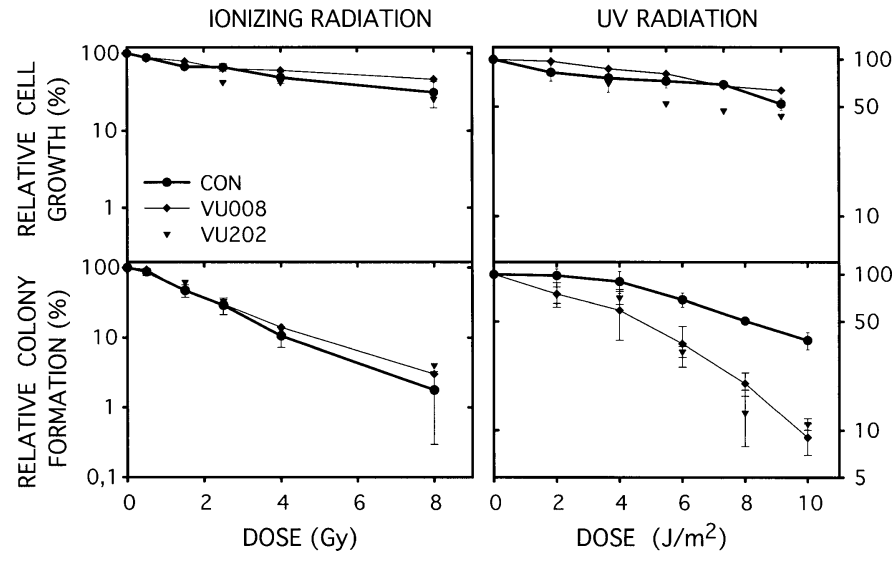

FIG. 3. Radiation-induced reductions of cell growth and colony formation of FA-D2 strains. Relative numbers of cells in fibroblast mass cultures (top panels) and relative numbers of colonies after dilute plating (bottom panels) after exposure of cells to increasing doses of ionizing radiation (left panels) and UV light (right panels). FA-D2, fibroblast cultures derived from patients belonging to complementation group D2. FAD2 strains are denoted by thin dotted or solid lines connecting inverted triangles and diamonds. The dark and solid lines connecting solid circles denote means $\pm 1 \mathrm{SD}$ of four control strains derived from healthy donors.

A close to normal UV-radiation response of FA cells is consistent with the fact that the major DNA lesion after UV irradiation consists of DNA adducts that are removed by nucleotide excision repair, a process that has been shown to function normally in FA cells (29). Nevertheless, it may be of interest that yeast two-hybrid studies have shown a much more prominent interaction between the products of FANCA and FANCG genes compared to other FA gene products (30) so that the common radiation response of these two complementation groups may reflect their close functional relationship.

Whereas our study provides no evidence for increased ionizing radiation sensitivity of FA-D2 primary fibroblast cultures, our two FA-D2 strains were found to be more UVradiation sensitive than strains from non-FA donors with regard to their colony-forming ability. It must be pointed out, however, that the UV-radiation-induced decrease in colony numbers in this group of strains is far less severe than that of cells with the CS genotype. To establish firmly whether the observed dose-dependent reduction in colony formation after UV-radiation exposure might be a consistent and characteristic property of FA-D2 cells, additional strains from different FA-D2 donors with other genetic backgrounds need to be tested. We clearly cannot exclude the possibility that the unusual behavior of our FA-D2 strains might be a special characteristic of the genetic background of these particular strains that were derived from sibling donors. Moreover, the question remains why the reduced colony survival of our FA-D2 strains after UV-radiation exposure was not paralleled by the corresponding cell growth data. We can only speculate that the 100-fold difference in plating density, by some mechanism such as metabolic cooperation and/or the bystander effect (31), may have contributed to these discrepant results.

A certain degree of variability among cell lines has been a common experience in similar studies and may reflect subtle differences in genetic background, growth fraction, and cell culture conditions. A case in point is the study by Carreau and colleagues (6), who tested the drug sensitivity spectra of FA lymphoblast lines and found a degree of variation similar to that described in the present study but few systematic differences. In particular, there was no difference in the UV-radiation response of lymphoblast cell lines originating from various complementation groups. However, Carreau et al. (6) found increased sensitivity of some of their cell lines to the radiomimetic drug bleomycin. It is clear that the response to clastogens can vary as a function of cell type. Differences in the radiosensitivity of FA lymphocytes and FA fibroblasts were noted in previous studies (32), and a recent study (33) describes consistent differences between FA fibroblasts and lymphoblasts in the way that the cells repair DNA double-strand breaks. In addition, FA lymphoblasts are known to undergo apoptosis effectively in response to DNA-crosslinking agents, whereas FA fibroblasts have been shown to be delayed in the $4 \mathrm{c}$ stage of the cell cycle and may eventually recover (34). In addition, it has not been determined whether the reported radiosensitivity of FA lymphoblast cell lines $(6,35)$ persists under hypoxic cell culture conditions. Since fibroblast cultures derived from FA knockout mice of the complementation groups $\mathrm{A}, \mathrm{C}$ and $\mathrm{G}$ are not radiosensitive (36), it appears that mesenchymal cell types from both mice and humans agree with respect to their in vitro response to ionizing radiation.

Radiation therapy studies generally report a positive correlation between in vivo and in vitro radiosensitivity (37). However, at present the risks of radiation therapy in FA patients are not clear (38). When testing the in vitro response of cells from patients clastogens, one must be aware of the fact that the (artificial) in vitro situation may not be representative of the (physiological) in vivo state. This complicates the "predictive" value of in vitro sensitivity assays. A very instructive case in point is the pronounced discrepancy between in vivo and in vitro radiosensitivity that has been reported by Marcou et al. (39). While their FA patient showed a severe tissue reaction during radiation therapy for tonsillar carcinoma, there was no evidence for in vitro radiosensitivity using conventional assays with cultures of the patient's fibroblasts. As mentioned above, it is conceivable that different cell types use different mechanisms to respond to DNA damage, which could account in part for differences when comparing in vivo with in vitro exposures.

In summary, with the possible exception of minor alterations in the UV-radiation response of FA-A, FA-G and FAD2 cells, our study provides no evidence for systematic differences in radiation response as a function of FA complementation group, nor do we find evidence for increased radiosensitivity of FA fibroblasts of any complementation 
group compared to fibroblasts from healthy donors. This is also true for the single FA-D1 strain included in our testing, which has recently been shown to carry biallelic mutations in the BRCA2 gene (40); this is true for the ionizing radiation response of our two strains belonging to complementation group FA-D2, which acts downstream of and divergent from the other members of the FA protein complex $(10,41)$. It must be stressed, however, that our results were obtained under hypoxic cell culture conditions, and using fibroblast strains with major proven ionizing radiation and UV-radiation sensitivities (AT and CS cells) as positive controls. Since each of the positive controls represents an extreme degree of sensitivity, minor degrees of sensitivity, such as observed with some of our UV-irradiated strains, cannot be excluded since such minor sensitivities cannot be reliably separated from the background variation of our cell culture assays.

\section{ACKNOWLEDGMENTS}

We are grateful to Kristina Drexler, who initially explored the technical conditions and performed preliminary experiments. We thank Michael Flentje, head of the Department of Radiation Therapy at the University of Wuerzburg, for ${ }^{60} \mathrm{Co} \gamma$ irradiations. We are indebted to the SchroederKurth-Fonds and the Deutsche Fanconi-Anaemie-Hilfe for financial support.

Received: July 25, 2003; accepted: November 14, 2003

\section{REFERENCES}

1. H. Joenje and K. J. Patel, The emerging genetic and molecular basis of Fanconi anaemia. Nat. Rev. Genet. 2, 446-457 (2001).

2. P. Guardiola, R. Pasquini, L. Dokal, J. J. Ortega, M. van Weel-Sipman, J. C. W. Marsh, S. E. Ball, F. Locatelli, C. Vermylen and E. Gluckman, Outcome of 69 allogeneic stem cell transplantations for Fanconi anemia using HLA-matched unrelated donors: A study on behalf of the European Group for Blood and Marrow Transplantation. Blood 95, 422-429 (2000).

3. C. S. Djuzenova, A. Rothfuss, U. Oppitz, G. Speit, D. Schindler, H. Hoehn and M. Flentje, Response to X-irradiation of Fanconi anemia homozygous and heterozygous cells assessed by the single-cell gel electrophoresis (comet) assay. Lab. Invest. 81, 185-192 (2001).

4. E. Gluckman, Radiosensitivity in Fanconi anemia: Application to the conditioning for bone marrow transplantation. Radiother. Oncol. 18 (Suppl. 1), 88-93 (1990).

5. M. L. MacMillan, A. D. Auerbach, S. M. Davies, T. E. DeFor, A. Gillio, R. Giller, R. Harris, M. Cairo, K. Dusenbery and J. E. Wagner, Haematopoietic cell transplantation in patients with Fanconi anaemia using alternate donors: Results of a total body irradiation dose escalation trial. Br. J. Haematol. 109, 121-129 (2000).

6. M. Carreau, N. Alon, L. Bosnoyan-Collins, H. Joenje and M. Buchwald, Drug sensitivity spectra in Fanconi anemia lymphoblastoid cell lines of defined complementation groups. Mutat. Res. 435, 103-109 (1999).

7. H. Joenje, M. Levitus, Q. Waisfisz, A. D'Andrea, I. Garcia-Higuera, T. Pearson, C. G. van Berkel, M. A. Rooimans, N. Morgan and F. Arwert, Complementation analysis in Fanconi anemia: Assignment of the reference FA-H patient to group A. Am. J. Hum. Genet. 67, 759-762 (2000).

8. D. B. Busch, M. Z. Zdzienicka, A. T. Natarajan, N. J. Jones, W. J. Overkamp, A. Collins, D. L. Mitchell, M. Stefanini, E. Botta and L. $\mathrm{H}$. Thompson, A CHO mutant, UV40, that is sensitive to diverse mutagens and represents a new complementation group of mitomycin C sensitivity. Mutat. Res. 363, 209-221 (1996).

9. J. B. Wilson, M. A. Johnson, A. P. Stuckert, K. L. Trueman, S. May, P. E. Bryant, R. E. Meyn, A. D. D'Andrea and N. J. Jones, The Chinese hamster FANCG/XRCC9 mutant NM3 fails to express the monoubiquinated form of the FANCD2 protein, is hypersensitive to a range of DNA damaging agents and exhibits a normal level of spontaneous sister chromatid exchange. Carcinogenesis 22, 19391946 (2001).

10. I. Garcia-Higuera, T. Taniguchi, S. Ganesan, M. S. Meyn, C. Timmers, J. Hejna, M. Grompe and A. D. D'Andrea, Interaction of the Fanconi anemia proteins and BRCA1 in a common pathway. Mol. Cell. 7, 249-262 (2001).

11. H. Joenje, F. Arwert, A. W. Eriksson, H. de Koning and A. B. Oostra, Oxygen-dependence of chromosomal aberrations in Fanconi's anaemia. Nature 290, 142-143 (1981).

12. D. Schindler and H. Hoehn, Fanconi anemia mutation causes cellular susceptibility to ambient oxygen. Am. J. Hum. Genet. 43, 429-435 (1988).

13. H. Joenje, A. B. Oostra, M. Wijker, F. M. di Summa, C. G. M. van Berkel, M. A. Rooimans, W. Ebell, L. van Weel, J. C. Pronk and F. Arwert, Evidence for at least eight Fanconi anemia genes. Am. J. Hum. Genet. 61, 940-924 (1997).

14. Fanconi Anemia Mutation Database, The Rockefeller University, http://rockefeller.edu/fanconi/mutate/default.html.

15. J. Pomp, J. L. Wike, I. J. M. Ouwerkerk, C. Hoogstraten, J. Davelaar, P. I. Schrier, J. W. H. Leer, H. D. Thames and W. A. Brock, Cell density dependent plating efficiency affects outcome and interpretation of colony forming assays. Radiother. Oncol. 40, 121-125 (1996).

16. J. B. Little, J. Nove, L. C. Strong and W. W. Nichols, Survival of human diploid skin fibroblasts from normal individuals after X-irradiation. Int. J. Radiat. Biol. 54, 899-910 (1988).

17. J. B. Little and J. Nove, Sensitivity of human diploid fibroblast cell strains from various genetic disorders to acute and protracted radiation exposure. Radiat. Res. 123, 87-92 (1990).

18. H. Nagasawa and J. B. Little, Radiosensitivities of ten apparently normal human diploid fibroblast strains to cell killing, $\mathrm{G}_{2}$-phase chromosomal aberrations, and cell cycle delay. Cancer Res. 48, 45354538 (1988).

19. S. Ma, R-H. Fang and W. P. Chang, Heterogeneity in radiosensitivity of human diploid fibroblasts from keloids and normal skins. Cell Biol. Int. 20, 289-292 (1996).

20. J. N. DeSimone, H. Dolezalova, J. L. Redpath and E. J. Stanbridge, Prolonged cell cycle arrest in irradiated human diploid skin fibroblasts: The role of nutrient deprivation. Radiat. Res. 153, 131-143 (2000).

21. L. N. Su and J. B. Little, Transformation and radiosensitivity of human diploid skin fibroblasts transfected with activated ras oncogene and SV40 T-antigen. Int. J. Radiat. Biol. 62, 201-210 (1992).

22. L. N. Su and J. B. Little, Transformation and radiosensitivity of human diploid skin fibroblasts transfected with SV40 T-antigen mutants defective in RB and P53 binding domains. Int. J. Radiat. Biol. 62, 461-468 (1992).

23. N. M. Tsang, H. Nagasawa, C. Li and J. B. Little, Abrogation of p53 function by transfection of HPV16 E6 gene enhances the resistance of human diploid fibroblasts to ionizing radiation. Oncogene 10, 2403-2408 (1995).

24. B. L. Kuehl, C. B. Brezden, R. D. Traver, D. Siegel, D. Ross, J. Renzing and A. M. Rauth, Immortalisation of a human diploid fibroblast cell strain: A DT-diaphorase paradox. Br. J. Cancer 27 (Suppl.), S19-S22 (1996).

25. H. Saito, A. T. Hammond and R. E. Moses, Hypersensitivity to oxygen is a uniform and secondary defect in Fanconi anemia cells. Mutat. Res. 294, 255-262 (1993).

26. M. Futaki, T. Igarashi, S. Watanabe, S. Kajigaya, A. Tatsuguchi, J. Wang and J. M. Liu, The FANCG Fanconi anemia protein interacts with CYP2E1: Possible role in protection against oxidative DNA damage. Carcinogenesis 23, 67-72 (2002). 
27. R. Iyer and B. E. Lehnert, Effects of ionizing radiation in targeted and nontargeted cells. Arch. Biochem. Biophys. 376, 14-25 (2000).

28. J. P. de Winter, Q. Waisfisz, M. A. Rooimans, C. G. M. van Berkel, L. Bosnoyan-Collins, N. Alon, M. Carreau, O. Bender, I. Demuth and $\mathrm{H}$. Joenje, The Fanconi anaemia group G gene FANCG is identical with XRCC9. Nat. Genet. 20, 281-283 (1998).

29. J. Hansson, S. M. Keyse, T. Lindahl and R. D. Wood, DNA excision repair in cell extracts from human cell lines exhibiting hypersensitivity to DNA-damaging agents. Cancer Res. 51, 3384-3390 (1991).

30. T. Reuter, S. Herterich, O. Bernhard, H. Hoehn and H. J. Gross, Strong FANCA/FANCG but weak FANCA/FANCC interaction in the yeast 2-hybrid system. Blood 95, 719-720 (2000).

31. J. F. Ward, The radiation-induced lesions which trigger the bystander effect. Mutat. Res. 499, 151-154 (2002).

32. G. Duckworth-Rysiecki and A. M. Taylor, Effects of ionizing radiation on cells from Fanconi's anemia patients. Cancer Res. 45, 416420 (1985).

33. S. L. Donahue, R. Lundberg and C. Campbell, Deficient in vivo DNA end-joining in Fanconi anemia fibroblasts. Am. J. Hum. Genet. 69 (Suppl.), 216 (2001).

34. Y. M. N. Akkari, R. L. Bateman, C. A. Reifsteck, A. D. D'Andrea, S. B. Olsen and M. Grompe, The 4N cell cycle delay in Fanconi anemia reflects growth arrest in late S phase. Mol. Genet. Metabol. 74, 403-412 (2001).
35. R. A. Gatti, The inherited basis of human radiosensitivity. Acta Oncol. 40, 702-711 (2001)

36. M. Koomen, N. C. Cheng, H. J. van de Vrugt, B. C. Godthelp, M. A. van der Valk, A. B. Oostra, M. Z. Zdzienicka, H. Joenje and F. Arwert, Reduced fertility and hypersensitivity to mitomycin C characterize Fancg/Xrcc9 null mice. Hum. Mol. Genet. 11, 273-281 (2002).

37. J. S. Loeffler, J. R. Harris, W. K. Dahlberg and J. B. Little, In vitro radiosensitivity of human diploid fibroblasts derived from women with unusually sensitive clinical responses to definitive radiation therapy for breast cancer. Radiat. Res. 121, 227-231 (1990).

38. B. P. Alter, Radiosensitivity in Fanconi's anemia patients. Radiother. Oncol. 62, 345-347 (2002).

39. Y. Marcoua, A. D'Andrea, P. A. Jeggo and P. N. Plowman, Normal cellular radiosensitivity in an adult Fanconi anaemia patient with marked clinical radiosensitivity. Radiother. Oncol. 60, 75-79 (2001).

40. N. G. Howlett, T. Taniguchi, S. Olsen, B. Cox, Q. Waisfisz, C. de Die-Smulders, N. Persky, M. Grompe, H. Joenje and A. D. D'Andrea, Biallelic inactivation of BRCA2 in Fanconi anemia. Science 297, 606-609 (2002).

41. S. L. Donahue, R. Lundberg, R. Saplis and C. Campell, Deficient regulation of DNA double-strand break repair in Fanconi anemia fibroblasts. J. Biol. Chem. 278, 29487-29495 (2003). 